\title{
Time-evolution of magnetic field in hot nuclear matter with fluctuating topological charge
}

\author{
Kirill Tuchin (1) \\ Department of Physics and Astronomy, Iowa State University, Ames, Iowa 50011, USA
}

(Received 19 November 2019; accepted 11 May 2020; published 15 July 2020)

\begin{abstract}
The time-evolution of the magnetic field in hot homogeneous nuclear matter has two qualitatively different stages separated by the sphaleron transition time $\tau_{c}$. At early times, when the chiral conductivity $\sigma_{\chi}$ is a slow function of time, the soft chiral modes $k<\sigma_{\chi}$ of the magnetic field grow exponentially with time, which is known as the chiral instability. At later times $\sigma_{\chi}$ fluctuates due to the sphaleron transitions and can be regarded as a random process. It is argued that the average magnetic field is exponentially damped at later times. The time-evolution of the average field energy is more complicated and depends on the electrical conductivity of the chiral matter but does not depend on chirality. It exhibits instability only if the matter is a poor electrical conductor, such as the quark-gluon plasma near the critical temperature. The precise conditions for the instability and the growth rate of the unstable modes are derived.
\end{abstract}

DOI: 10.1103/PhysRevC.102.014908

\section{INTRODUCTION}

The topological configurations of gluon fields form $P$ and $T$-odd domains in hot nuclear matter. Their topological charge couples to the electromagnetic fields by way of the chiral anomaly. Electrodynamics, modified in the presence of the topological domains, exhibits a number of novel effects such as the chiral magnetic effect $[1,2]$ and the anomalous Hall effect [3]. One of its features that attracted a considerable interest is the emergence of the soft magnetic field modes exponentially growing in time known as the chiral instability [4-19]. These unstable modes transfer helicity from the chiral medium, such as the quark-gluon plasma, to the magnetic field in a process known as the inverse cascade $[4,20]$.

The chiral instability can be most easily derived using the effective Maxwell-Chern-Simons theory which adds to the Maxwell Lagrangian a term that couples $F \tilde{F}$ directly to the topological domains by means of the chiral anomaly [21-23]. The dynamics of the electromagnetic field in the spatially uniform chiral matter with electrical conductivity $\sigma$ can be described by the vector potential $\boldsymbol{A}$ which satisfies the following equation in the radiation gauge

$$
-\nabla^{2} \boldsymbol{A}=-\partial_{t}^{2} \boldsymbol{A}-\sigma \partial_{t} \boldsymbol{A}+\sigma_{\chi}(t) \boldsymbol{\nabla} \times \boldsymbol{A},
$$

where $\sigma_{\chi}$ is the chiral conductivity [2,24] sourced by the topological gluon field configurations, see the Appendix for more details. The ratio $\mu_{5}=\sigma_{\chi} / c_{A}$, where $c_{A}$ is the anomaly

Published by the American Physical Society under the terms of the Creative Commons Attribution 4.0 International license. Further distribution of this work must maintain attribution to the author(s) and the published article's title, journal citation, and DOI. Funded by $S C O A P^{3}$. coefficient, is also used throughout the paper. ${ }^{1}$ Seeking a solution in the form $\boldsymbol{A}=a_{\boldsymbol{k} \lambda}(t) \boldsymbol{\epsilon}_{\lambda} e^{i \boldsymbol{k} \cdot \boldsymbol{x}}$, where $\boldsymbol{\epsilon}_{\lambda}$ are the circular polarization vectors of helicity $\lambda= \pm 1$ and using the identity $i \boldsymbol{k} \times \boldsymbol{\epsilon}_{\lambda}=k \lambda \boldsymbol{\epsilon}_{\lambda}$ one deduces that the amplitude $a_{\boldsymbol{k} \lambda}$ satisfies the equation

$$
\ddot{a}_{k \lambda}+\sigma \dot{a}_{k \lambda}+k\left(k-\lambda \sigma_{\chi}\right) a_{k \lambda}=0 .
$$

Assuming that the chiral conductivity is a slow function of time and neglecting for the sake of brevity the electrical conductivity, the magnetic field for each plane wave mode reads, up to the pre-exponential factors,

$$
\boldsymbol{B} \sim e^{i k \cdot \boldsymbol{x}} \exp \left\{\mp i t \sqrt{k\left(k+\lambda \sigma_{\chi}\right)}\right\} .
$$

Clearly, when $\lambda \sigma_{\chi}<0$ the magnetic field modes $k<\left|\lambda \sigma_{\chi}\right|$ are unstable [5]. This instability is manifestly chiral.

It is remarkable, that since Eq. (3) satisfies the modified Maxwell equations, the exponential growth of the magnetic field is not constrained by the energy conservation [17,29]. However, it is constrained by the conservation of the total helicity of the magnetic field and the chiral matter, which follows from the chiral anomaly equation [26,27]. As the magnetic field increases, the helicity flows from the chiral matter to the field until the chiral conductivity vanishes $[4,17,28,29]$. As argued in [29], this helicity transfer is an adiabatic process, meaning that $\sigma_{\chi}$ is a slowly varying function of time.

Let us now examine the assumptions that led us from a MCS equation (1) to the unstable solution (3). Dropping the first assumption $\sigma=0$ results in a more complicated expression for the magnetic field. However, the finite value of the electrical conductivity does not have any significant effect

\footnotetext{
${ }^{1}$ In models with fermions $\mu_{5}$ can be identified as the axial chemical potential. However, its physical interpretation is not free of difficulties, see [25] and references therein.
} 
upon the instability dynamics at constant (or slowly varying) $\sigma_{\chi}$ [29]. The second and crucial assumption is the slow variation of the chiral conductivity. It holds while $t<\tau_{c}$ where $\tau_{c}$ is the sphaleron transition time, i.e. the transition time between the gauge field configurations of different topological charge. By the time $t=\tau_{c}$, the exponent in Eq. (3) reaches its maximal value $\exp \left(\sigma_{\chi} \tau_{c}\right)$. The estimates of the sphaleron transition time $\tau_{c} \sim 1 /\left(g^{4} T\right)$ [30] and the chiral conductivity $\sigma_{\chi} \sim e^{2} \mu_{5}[2,24]$, at temperature $T$, indicate that $\sigma_{\chi} \tau_{c} \ll 1$. $^{2}$ The implication is that during the time interval $t<\tau_{c}$ when the chiral conductivity (and the corresponding topological charge density) may be regarded as slowly varying functions of time, the magnetic field instability simply does not have enough time to grow. Thus, in order to study the magnetic field instability in the hot nuclear matter, one is required to examine the opposite limit of $t \gg \tau_{c}$, when many sphaleron transition can occur causing fluctuations of the topological charge of the chiral domains.

The main goal of this paper is to examine this limit by regarding the chiral conductivity as a stochastic process with vanishing expectation value $\left\langle\sigma_{\chi}\right\rangle=0$. I assume that its dispersion $\Sigma_{\chi}=\sqrt{\left\langle\sigma_{\chi}^{2}\right\rangle}$ equals $c_{A} \mu_{5}$ and the autocorrelation function $\left\langle\sigma_{\chi}(t) \sigma_{\chi}(t-\tau)\right\rangle$ has support at $t<\tau_{c}$, meaning that the sphaleron transition time $\tau_{c}$ is the correlation time. Equation (2) then describes the harmonic oscillator with a random frequency. Using the Van Kampen's theory [33] one can deduce from Eq. (2) the ordinary differential equations for the average values of the field amplitude $\left\langle a_{k \lambda}\right\rangle$ and its second moments $\left\langle\left(a_{k \lambda}\right)^{2}\right\rangle,\left\langle\left(\dot{a}_{k \lambda}\right)^{2}\right\rangle$. The main result of this theory is that the average value of the magnetic field decays exponentially with time. The average value of the magnetic field energy also decays if the plasma is a good electric conductor, which occurs at high temperatures. However, at temperatures close to the critical temperature the plasma is a poor conductor and the average energy is unstable.

An important feature of the chiral instability is its remarkable universality in many chiral systems. The universality emerges in the adiabatic limit, when the details of the topological charge distribution are irrelevant, so that, for example, electromagnetic plasma with chirality imbalance and the quark-gluon plasma with a topological gluon field configuration have similar chiral instability, described by one parameter-the chiral conductivity, see [4-19] and references therein. This paper extends analysis of the chiral instability to systems whose topological charge fluctuations are stochastic in the long time limit. This approach is adequate for hot nuclear matter since the topological charge is determined mostly by the strong interactions, but may not universally hold in other chiral systems.

The paper is structured as follows. In Sec. II B the Van Kampen theory is reviewed and employed to reduce the stochastic equation (2) to the ordinary differential equations

\footnotetext{
${ }^{2}$ This holds true since $e^{2} / g^{4} \ll 1$ at any reasonable temperature and $\mu_{5}$ is expected to be of the order of $T$ or smaller. A more accurate estimate for the sphaleron transition time is $\tau_{c} \sim 1 /\left(g^{4} T\right) \ln g^{-1}$ $[31,32]$.
}

for the averages of the amplitude and its second moment. The solutions to these equations are used in Secs. III and IV to analyze the time evolution of the average magnetic field, electromagnetic energy and magnetic helicity with time. The Summary and Discussion is presented in Sec. V.

\section{EVOLUTION EQUATIONS FOR THE AMPLITUDE MOMENTS}

To study the late time behavior of the magnetic field, one can regard the chiral conductivity $\sigma_{\chi}(t)$ as a random process and hence Eq. (2) becomes a stochastic equation describing time-evolution of the field amplitude with momentum $\boldsymbol{k}$ and polarization $\lambda$. Equation (2) does not have an analytical solution. However, one can deduce from it ordinary differential equations for the expectation value of the amplitude moments using the Van Kampen theory [33]. This section represents a brief summary of the relevant results. For the reasons explained below, the underdamped and the overdamped modes with $k \gtrsim \sigma / 2$ and $k \lesssim \sigma / 2$, respectively, are considered separately from the critically damped ones with $k \approx \sigma / 2$.

\section{A. Underdamped and overdamped modes}

To begin with, I define, for a given $\boldsymbol{k}$ and $\lambda$, a new variable $x=a_{k \lambda} e^{\sigma t / 2}$ which satisfies the equation

$$
\ddot{x}(t)+\omega^{2}[1+\alpha \xi(t)] x(t)=0,
$$

where

$$
\begin{aligned}
\omega^{2} & =k^{2}-\frac{\sigma^{2}}{4}, \quad \alpha=-\frac{\lambda k}{\omega^{2}} \Sigma_{\chi}, \quad \xi=\frac{\sigma_{\chi}}{\Sigma_{\chi}}, \\
\Sigma_{\chi} & =\sqrt{\left\langle\sigma_{\chi}^{2}\right\rangle} .
\end{aligned}
$$

The random process $\xi(t)$ is such that $\langle\xi\rangle=0$. The correlation time of $\xi$ is $\tau_{c}$, meaning that the autocorrelation function $\langle\xi(t) \xi(t-\tau)\rangle$ vanishes at $\tau>\tau_{c}$. For the modes with $\omega \neq 0$ it is convenient to introduce the dimensionless time variable $t^{\prime}=\omega t$.

Equation (4) can be cast in the matrix form

$$
\frac{d u\left(t^{\prime}\right)}{d t^{\prime}}=\left[A_{0}+\alpha \xi\left(t^{\prime}\right) B\right] u\left(t^{\prime}\right),
$$

where $u=\left(x, x^{\prime}\right)^{T}$ with $x^{\prime}=d x / d t^{\prime}$ and

$$
A_{0}=\left(\begin{array}{cc}
0 & 1 \\
-1 & 0
\end{array}\right), \quad B=\left(\begin{array}{cc}
0 & 0 \\
-1 & 0
\end{array}\right) \text {. }
$$

The main result of [33] is that given Eq. (6) with arbitrary $u, A_{0}$, and $B$, at late times $t \gg \tau_{c}$ the expectation value of $u$ satisfies the equation

$$
\begin{aligned}
\frac{d\left\langle u\left(t^{\prime}\right)\right\rangle}{d t^{\prime}}= & \left\{A_{0}+\alpha^{2} \int_{0}^{\infty}\left\langle\xi\left(t^{\prime}\right) \xi\left(t^{\prime}-\tau^{\prime}\right)\right\rangle B e^{A_{0} \tau^{\prime}} B e^{-A_{0} \tau^{\prime}} d \tau^{\prime}\right\} \\
& \times\left\langle u\left(t^{\prime}\right)\right\rangle,
\end{aligned}
$$

provided that

$$
|\alpha \omega| \tau_{c} \ll 1
$$


which allows treating the fluctuating term in Eq. (4) as a perturbation. In view of $\omega$-dependence of $\alpha$ in Eq. (5), the condition (9) excludes modes with small $\omega$ 's. Namely, expanding $k=\sigma / 2+\delta k$ one obtains that Eq. (9) is satisfied if

$$
|\delta k| \gg \frac{\sigma}{4}\left(\Sigma_{\chi} \tau_{c}\right)^{2}
$$

Since $\Sigma_{\chi} \tau_{c} \sim e^{2} \mu_{5} / g^{4} T \ll 1$, Eq. (9) holds for all $k$ 's except in the narrow interval around $k=\sigma / 2$. The modes with $\delta k \lesssim \sigma\left(\Sigma_{\chi} \tau_{c} / 2\right)^{2}$ will be referred to as the critically dumped modes, while those satisfying Eq. (10) with $\delta k>0$ and $\delta k<$ 0 as underdamped and overdamped modes, respectively.

Equation (8) can now be applied to the underdamped and overdamped modes. Computing with the help of Eq. (7)

$$
B e^{A_{0} \tau^{\prime}} B e^{-A_{0} \tau^{\prime}}=\left(\begin{array}{cc}
0 & 0 \\
\sin \tau^{\prime} \cos \tau^{\prime} & -\sin ^{2} \tau^{\prime}
\end{array}\right),
$$

substituting into Eq. (8) and converting the result into the second order differential equation for $\langle x\rangle$ yields

$$
\frac{d^{2}\langle x\rangle}{d t^{\prime 2}}+\frac{1}{2} \alpha^{2} c_{2} \frac{d\langle x\rangle}{d t^{\prime}}+\left(1-\frac{1}{2} \alpha^{2} c_{1}\right)\langle x\rangle=0
$$

where

$$
\begin{aligned}
& c_{1}=\int_{0}^{\infty}\left\langle\xi\left(t^{\prime}\right) \xi\left(t^{\prime}-\tau^{\prime}\right)\right\rangle \sin \left(2 \tau^{\prime}\right) d \tau^{\prime}, \\
& c_{2}=\int_{0}^{\infty}\left\langle\xi\left(t^{\prime}\right) \xi\left(t^{\prime}-\tau^{\prime}\right)\right\rangle\left[1-\cos \left(2 \tau^{\prime}\right)\right] d \tau^{\prime} .
\end{aligned}
$$

The evolution equations for the second moments of $x$ can be obtained by applying Eqs. (6),(8) to the vector $u=$ $\left(x, x^{\prime 2}, x x^{\prime}\right)^{T}$. The corresponding matrices are

$$
A_{0}=\left(\begin{array}{ccc}
0 & 0 & 2 \\
0 & 0 & -2 \\
-1 & 1 & 0
\end{array}\right), \quad B=\left(\begin{array}{ccc}
0 & 0 & 0 \\
0 & 0 & -2 \\
-1 & 0 & 0
\end{array}\right)
$$

which produces

$$
B e^{A_{0} \tau^{\prime}} B e^{-A_{0} \tau^{\prime}}=\left(\begin{array}{ccc}
0 & 0 & 0 \\
2 \cos ^{2} \tau^{\prime} & -2 \sin ^{2} \tau^{\prime} & 0 \\
\sin 2 \tau^{\prime} & 0 & -2 \sin ^{2} \tau^{\prime}
\end{array}\right) .
$$

Equation (8) now reads

$$
\frac{d}{d t^{\prime}}\left(\begin{array}{c}
\left\langle x^{2}\right\rangle \\
\left\langle x^{\prime 2}\right\rangle \\
\left\langle x x^{\prime}\right\rangle
\end{array}\right)=\left(\begin{array}{ccc}
0 & 0 & 2 \\
\alpha^{2} c_{3} & -\alpha^{2} c_{2} & -2 \\
-1+\alpha^{2} c_{1} & 1 & -\alpha^{2} c_{2}
\end{array}\right)\left(\begin{array}{c}
\left\langle x^{2}\right\rangle \\
\left\langle x^{\prime 2}\right\rangle \\
\left\langle x x^{\prime}\right\rangle
\end{array}\right),
$$

where

$$
c_{3}=\int_{0}^{\infty}\left\langle\xi\left(t^{\prime}\right) \xi\left(t^{\prime}-\tau^{\prime}\right)\right\rangle\left(1+\cos \left(2 \tau^{\prime}\right)\right) d \tau^{\prime} .
$$

\section{B. Critically damped modes}

In general, the Van Kampen theory cannot be applied to all critically damped modes because $\omega^{2}$ is not negligible as compared to the fluctuating term $\lambda k \Sigma_{\chi} \xi$ in Eq. (4). The exception is the mode with momentum $k=\sigma / 2$ in which case case $\omega=0$ and Eq. (4) takes form

$$
\ddot{x}(t)+\beta^{2} \xi(t) x(t)=0,
$$

where $\xi$ is defined as in Eq. (5) and

$$
\beta^{2}=-\Sigma_{\chi} \lambda k=-\frac{1}{2} \Sigma_{\chi} \lambda \sigma .
$$

Passing to the dimensionless time variable $t^{\prime}=\beta t$, one can write Eq. (18) in the form (6) with $\alpha=1$ and

$$
A_{0}=\left(\begin{array}{ll}
0 & 1 \\
0 & 0
\end{array}\right), \quad B=\left(\begin{array}{cc}
0 & 0 \\
-1 & 0
\end{array}\right),
$$

which implies

$$
B e^{A_{0} \tau^{\prime}} B e^{-A_{0} \tau^{\prime}}=\left(\begin{array}{cc}
0 & 0 \\
\tau^{\prime} & -\tau^{\prime 2}
\end{array}\right) .
$$

The fluctuating term is a small perturbation of the initial condition if $|\beta| \tau_{c} \ll 1$. In this case, at $t \gg \tau_{c}$ the expectation value of $u=\left(x, x^{\prime}\right)^{T}$ obeys Eq. (8) with $\alpha=1$. Thus, Eq. (12) with $\alpha=1$ and $t^{\prime}=\beta t$ can be used to describe the time evolution of $\omega=0$ mode. The corresponding $c$ coefficients read

$$
\begin{aligned}
& c_{1}=2 \int_{0}^{\infty}\left\langle\xi\left(t^{\prime}\right) \xi\left(t^{\prime}-\tau^{\prime}\right)\right\rangle \tau^{\prime} d \tau^{\prime}, \\
& c_{2}=2 \int_{0}^{\infty}\left\langle\xi\left(t^{\prime}\right) \xi\left(t^{\prime}-\tau^{\prime}\right)\right\rangle \tau^{\prime 2} d \tau^{\prime} .
\end{aligned}
$$

Similarly, $u=\left(x^{2}, x^{\prime 2}, x x^{\prime}\right)^{T}$ obeys Eq. (6) with

$$
A_{0}=\left(\begin{array}{lll}
0 & 0 & 2 \\
0 & 0 & 0 \\
0 & 1 & 0
\end{array}\right), \quad B=\left(\begin{array}{ccc}
0 & 0 & 0 \\
0 & 0 & -2 \\
-1 & 0 & 0
\end{array}\right)
$$

and

$$
B e^{A_{0} \tau^{\prime}} B e^{-A_{0} \tau^{\prime}}=2\left(\begin{array}{ccc}
0 & 0 & 0 \\
1 & -\tau^{\prime 2} & 0 \\
\tau^{\prime} & 0 & -2 \tau^{\prime 2}
\end{array}\right)
$$

The corresponding equations for the second moments has the same form as Eq. (16) with $\alpha=1$, and $c$ coefficients given by Eqs. (22a),(22b), and

$$
c_{3}=2 \int_{0}^{\infty}\left\langle\xi\left(t^{\prime}\right) \xi\left(t^{\prime}-\tau^{\prime}\right)\right\rangle\left(1-\tau^{\prime 2}\right) d \tau^{\prime} .
$$

\section{Coefficients $c_{1}, c_{2}, c_{3}$}

It is useful for future reference to estimate the coefficients $c_{1}, c_{2}, c_{3}$ appearing in the evolution equations in the previous subsections. Their $\omega$-dependence is qualitatively different for good and poor electric conductors. The hot nuclear matter is a good electric conductor at high temperatures. Its electrical conductivity is of the order $\sigma \sim T / e^{2}{ }^{3}$ However, at temperatures above but close to the critical temperature the hot nuclear matter is a poor conductor with the electrical conductivity $\sigma \sim e^{2} T$ [35-38].

\footnotetext{
${ }^{3}$ More precisely $\sigma=13 T / e^{2} \ln e^{-1}$ [34].
} 


\section{Well conducting chiral matter}

(a) Consider the underdamped and overdamped modes satisfying Eq. (10), which excludes modes in a vicinity of $k=\sigma / 2$. In both cases $|\omega| \tau_{c}>\sqrt{\sigma \delta k} \tau_{c} \gg$ $\sigma \Sigma_{\chi} \tau_{c}^{2} \sim \mu_{5} / g^{8} T \gg 1$ for the electrical conductivity $\sigma \sim T / e^{2}$. Since the autocorrelation function equals one at $\tau \ll \tau_{c}$ and vanishes at $\tau>\tau_{c}$ one can estimate Eqs. (13), (17) as

$$
\begin{aligned}
& c_{1}=\omega \int_{0}^{\infty}\langle\xi(t) \xi(t-\tau)\rangle \sin (2 \omega \tau) d \tau \sim \omega \int_{0}^{1 / 2 \omega} 2 \omega \tau d \tau=\frac{1}{4}, \\
& c_{2}=2 \omega \int_{0}^{\infty}\langle\xi(t) \xi(t-\tau)\rangle \sin ^{2}(\omega \tau) d \tau \sim \omega \int_{0}^{\tau_{c}} d \tau=\omega \tau_{c} \\
& c_{3}=2 \omega \int_{0}^{\infty}\langle\xi(t) \xi(t-\tau)\rangle \cos ^{2}(\omega \tau) d \tau=c_{2}+\mathcal{O}\left(1 / \omega \tau_{c}\right) .
\end{aligned}
$$

This implies that $c_{2} \approx c_{3} \gg c_{1}$.

The coefficient $c_{2}$ may become negative if the spectrum of $\xi$ contains a mode oscillating with frequency $2 \omega$. Indeed the contribution of such mode to $c_{2}$ can be estimated as

$$
\begin{aligned}
\Delta c_{2} & \propto \omega \int_{0}^{\infty}\langle\xi(t) \xi(t-\tau)\rangle[1-\cos (2 \omega \tau)] \cos (2 \omega \tau) d \tau \\
& \sim-\frac{\omega}{2} \int_{0}^{\tau_{c}} d \tau=-\omega \tau_{c} .
\end{aligned}
$$

This causes the parametric resonance, i.e. the exponential growth of $\langle x\rangle$ with time, see Eq. (32) below.

As a specific example, consider the OrnsteinUhlenbeck random process with the autocorrelation function

$$
\langle\xi(t) \xi(t-\tau)\rangle=e^{-\tau / \tau_{c}} .
$$

The corresponding coefficients read

$$
\begin{aligned}
& c_{1}=\frac{2\left(\omega \tau_{c}\right)^{2}}{1+4\left(\omega \tau_{c}\right)^{2}}, \quad c_{2}=\frac{4\left(\omega \tau_{c}\right)^{3}}{1+4\left(\omega \tau_{c}\right)^{2}}, \\
& c_{3}=\frac{\left[2+4\left(\omega \tau_{c}\right)^{2}\right]\left(\omega \tau_{c}\right)}{1+4\left(\omega \tau_{c}\right)^{2}} .
\end{aligned}
$$

Multiplying Eq. (28) by $\cos (2 \omega \tau)$ one can check that $c_{1} \rightarrow 1 / 8, c_{2} \rightarrow-\omega \tau_{c} / 2$, and $c_{3} \rightarrow \omega \tau_{c} / 2$ at $\omega \tau_{c} \gg$ 1. In the next subsection it is shown that at small $|\omega| \tau_{c}$, the coefficient $c_{2}$ is always positive and so there is no parametric resonance.

(b) The Van Kampen theory fails to describe the critically damped modes of the well-conducting plasma because $|\beta| \tau_{c} \sim \sqrt{\mu_{5} / T} / g^{4} \gg 1$ (unless $\mu_{5}$ is very small).

\section{Poorly conducting chiral matter}

(a) The parameter $\omega \tau_{c}$ of the underdamped modes $k \gg$ $\sigma / 2$ of a poorly conducting plasma can be estimated as $\omega \tau_{c} \sim k / g^{4} T$. The coefficients of the modes with $k \gg g^{4} T$ are given by Eq. (26), while the ones with $k \ll g^{4} T$ are estimated from Eqs. (13) and (17) as

$$
c_{1} \sim\left(\omega \tau_{c}\right)^{2}, \quad c_{2} \sim\left(\omega \tau_{c}\right)^{3}, \quad c_{3} \sim \omega \tau_{c} .
$$

Estimates 30 apply also to the overdamped modes $k \ll \sigma / 2$ because $|\omega| \tau_{c}<\sigma \tau_{c} / 2 \sim e^{2} / g^{4} \ll 1$. Inci- dentally, this implies that the coefficients 30 are strongly ordered as $c_{2} \ll c_{1} \ll c_{3} \ll 1$.

(b) The $c$ coefficients for the critically damped mode $k=$ $\sigma / 2$ can be estimated using Eqs. (13), (17) with the result similar to Eq. 30:

$$
c_{1} \sim\left(\beta \tau_{c}\right)^{2}, \quad c_{2} \sim\left(\beta \tau_{c}\right)^{3}, \quad c_{3} \sim \beta \tau_{c} .
$$

In contrast to the well conducting matter, the Van Kampen theory is valid in this case since $|\beta| \tau_{c} \sim$ $\left(e^{2} / g^{4}\right) \sqrt{\mu_{5} / T} \ll 1$.

\section{TIME-EVOLUTION OF AVERAGE MAGNETIC FIELD}

In this and the following sections the results of the previous section are employed to analyze the late-time behavior of the average magnetic field and average field energy.

The general solution of Eq. (12) is a linear combination of the functions

$$
\langle x(t)\rangle_{ \pm}=\exp \left\{ \pm i \omega t-\frac{\alpha^{2}}{4}\left(c_{2} \pm i c_{1}\right) \omega t\right\},
$$

where only terms of the order $\alpha^{2}$ are retained. The corresponding magnetic field amplitudes are

$$
\left\langle a_{k \lambda}(t)\right\rangle_{ \pm}=\exp \left\{ \pm i \omega t-\frac{\alpha^{2}}{4}\left(c_{2} \pm i c_{1}\right) \omega t-\frac{1}{2} \sigma t\right\} .
$$

\section{A. The underdamped modes $k>\sigma / 2$}

The condition for the instability of the underdamped modes

$$
-\frac{\alpha^{2}}{4} c_{2} \omega-\frac{\sigma}{2}>0
$$

can be satisfied only for the parametric resonance modes with $c_{2}<0$. Using the definition of $\alpha$ from Eq. (5) this yields

$$
k^{2} \Sigma_{\chi}^{2}\left|c_{2}\right|>2 \omega^{3} \sigma \text {. }
$$

As mentioned below Eq. (29), the parametric resonance occurs in good conductors at $\omega \tau_{c} \gtrsim 1$. Using the estimate (27) in Eq. (35) and noting that the best chance to satisfy it is at smaller $\omega$, expand it near $k=\sigma / 2$ to obtain $\delta k<\Sigma_{\chi}^{2} \tau_{c}$. On the other hand, $\omega \gtrsim 1 / \tau_{c}$ implies that $\delta k \gtrsim 1 / \sigma \tau_{c}^{2}$. The two conditions contradict each other since $\Sigma_{\chi}^{2} \sigma \tau_{c}^{3}<1$. Thus, there are no unstable underdamped modes of the average 
magnetic field. Even the parametric resonance mode in a well-conducting chiral matter is rapidly diffused due to the large electric conductivity.

\section{B. The overdamped modes $k<\sigma / 2$}

The amplitude of the overdamped modes can be written down as a linear combination of the functions

$$
\left\langle a_{k \lambda}(t)\right\rangle_{ \pm}=\exp \left\{\mp|\omega| t-\frac{i k^{2} \Sigma_{\chi}^{2}}{4 \omega^{4}}\left(c_{2} \pm i c_{1}\right)|\omega| t-\frac{1}{2} \sigma t\right\} .
$$

The second term in the exponent is a small correction to the first one. Clearly, $\left\langle a_{k \lambda}(t)\right\rangle_{+}$is stable. In $\left\langle a_{k \lambda}(t)\right\rangle_{-}$the first and the third terms in the exponent cancel out at $k \ll \sigma / 2$. Expanding to the second order in $k / \sigma$ yields

$$
\left\langle a_{k \lambda}(t)\right\rangle_{-}=\exp \left\{-\frac{k^{2} t}{\sigma}-\frac{k^{2} \Sigma_{\chi}^{2} \sigma t\left(i c_{2}+c_{1}\right)}{8 \omega^{4}}\right\} .
$$

Thus, the overdamped modes are stable at any conductivity.

\section{The critically damped mode $k=\sigma / 2$}

It remains to examine the critically damped mode $\omega=0$. As noted below Eqs. (29) and 31 only poor conductors can be consistently considered in the present framework. As shown in Sec. II B, the average amplitude can be obtained from Eq. (33) by replacing $\omega \rightarrow \beta$ and $\alpha \rightarrow 1$ :

$$
\left\langle a_{k \lambda}(t)\right\rangle_{ \pm}=\exp \left\{ \pm i \beta t-\frac{1}{4}\left(c_{2} \pm i c_{1}\right) \beta t-\frac{1}{2} \sigma t\right\} .
$$

The amplitude (38) of the critically damped mode exponentially decays at late time because $c_{2}>0$ as indicated by Eq. 31.

\section{Conclusion}

It follows from the analysis in this section that all magnetic field modes are stable at long times $t \gg \tau_{c}$. This of course also includes the modes with $k<\sigma_{\chi} / 2$ that exhibit exponential growth at early times.

\section{TIME-EVOLUTION OF MAGNETIC FIELD ENERGY AND HELICITY}

Having examined the time-evolution of the magnetic field which is determined by the first moment of the amplitudes, we turn to the evolution of the electromagnetic energy and magnetic helicity which is determined by the second moments. The electromagnetic field energy and magnetic helicity read

$$
\mathcal{E}=\frac{1}{2} \int\left(\boldsymbol{E}^{2}+\boldsymbol{B}^{2}\right) d^{3} x=\sum_{\boldsymbol{k}, \lambda} \mathcal{E}_{\boldsymbol{k} \lambda}=\sum_{\boldsymbol{k}, \lambda} \frac{1}{2 k}\left(\dot{a}_{\boldsymbol{k} \lambda}^{2}+a_{\boldsymbol{k} \lambda}^{2} k^{2}\right)
$$

$\mathcal{H}=\int \boldsymbol{A} \cdot \boldsymbol{B} d^{3} x=\sum_{\boldsymbol{k}, \lambda} \mathcal{H}_{\boldsymbol{k} \lambda}=\sum_{\boldsymbol{k}, \lambda} \lambda a_{\boldsymbol{k} \lambda}^{2}$,

where each mode with given $\boldsymbol{k}$ and $\lambda$ is normalized to one particle in a unit volume and the amplitudes $a_{k \lambda}$ are real.
The corresponding expressions for the expectation values of energy and helicity of each mode can be express in terms of the moments of the variable $x$ as

$$
\begin{aligned}
\left\langle\mathcal{E}_{\boldsymbol{k} \lambda}\right\rangle & =\frac{1}{2 k} e^{-\sigma t}\left[\left\langle x^{2}\right\rangle\left(k^{2}+\frac{1}{4} \sigma^{2}\right)-\langle\dot{x} x\rangle \sigma+\left\langle\dot{x}^{2}\right\rangle\right], \\
\left\langle\mathcal{H}_{k \lambda}\right\rangle & =\lambda e^{-\sigma t}\left\langle x^{2}\right\rangle .
\end{aligned}
$$

The time evolution of the second moments of $x$ is given by Eq. (16). The eigenvalues of the matrix in Eq. (16) to the order $\alpha^{2}$ are

$$
\begin{aligned}
& v_{0}=\frac{1}{2} \alpha^{2}\left(c_{3}-c_{2}\right), \\
& v_{ \pm}= \pm 2 i\left(1-\alpha^{2} \frac{c_{1}}{4}\right)-\frac{1}{4} \alpha^{2}\left(c_{3}+3 c_{2}\right) .
\end{aligned}
$$

Transforming Eq. (16) to the diagonal form and integrating one finds

$$
\begin{aligned}
\omega^{2}\left\langle x^{2}\right\rangle+\left\langle\dot{x}^{2}\right\rangle & =u_{0} e^{\nu_{0} \omega t}, \\
\omega^{2}\left\langle x^{2}\right\rangle-\left\langle\dot{x}^{2}\right\rangle+2 i \omega\langle x \dot{x}\rangle & =2 u_{-} e^{\nu_{-} \omega t} \\
-\omega^{2}\left\langle x^{2}\right\rangle+\left\langle\dot{x}^{2}\right\rangle+2 i \omega\langle x \dot{x}\rangle & =2 u_{+} e^{\nu_{+} \omega t}
\end{aligned}
$$

where $u_{0}, u_{ \pm}$are constants. Since $\nu_{+}=v_{-}^{*}$ the second moments and hence the amplitudes are real if $u_{+}=-u_{-}^{*}$. The solution thus reads

$$
\begin{aligned}
& \left\langle x^{2}\right\rangle=\frac{1}{2}\left[u_{0} e^{\nu_{0} \omega t}-u_{+}\left(e^{\nu_{-} \omega t}+e^{\nu_{+} \omega t}\right)\right], \\
& \left\langle\dot{x}^{2}\right\rangle=\frac{\omega^{2}}{2}\left[u_{0} e^{\nu_{0} \omega t}+u_{+}\left(e^{\nu_{-} \omega t}+e^{\nu_{+} \omega t}\right)\right], \\
& \langle x \dot{x}\rangle=\frac{\omega u_{+}}{2 i}\left(e^{\nu_{+} \omega t}-e^{\nu_{-} \omega t}\right) .
\end{aligned}
$$

When plugged into Eqs. (41),(42) it yields the timeevolution of the average energy and magnetic helicity. I turn now to the analysis of the instabilities. Note, that the instabilities of Eq. (45) are different from those of Eq. (33).

\section{A. The underdamped modes $k>\sigma / 2$}

Consider the well conducting chiral matter. Examination of Eqs. (45) and (41) reveals that the only underdamped $\left(\omega^{2}>\right.$ $0)$ nonresonant $\left(c_{2}>0\right)$ modes that are potentially unstable are those that satisfy $\nu_{0} \omega>\sigma$, i.e. $\omega \alpha^{2}\left(c_{3}-c_{2}\right)>2 \sigma$. For a good conductor Eq. (26c) implies that $c_{3}-c_{2} \sim 1 / \omega \tau_{c}$, so that this condition can be rewritten as $k^{2} \Sigma_{\chi}^{2} / \omega^{4} \tau_{c} \sigma>1$. At small $\omega$ one can expand $k$ near $\sigma / 2$ to obtain $\delta k^{2}<\Sigma_{\chi}^{2} / \sigma \tau_{c}$. This condition, however, contradicts Eq. (10) indicating that no underdamped nonresonant mode of a good conductor is divergent.

At the parametric resonance $\left(c_{2}<0\right)$, the instability can arise when either $\nu_{0} \omega>\sigma$ or $\operatorname{Re} \nu_{ \pm} \omega>\sigma$. In the former case, using $c_{3}-c_{2} \sim \omega \tau_{c}$ and repeating the arguments of the previous paragraph yields $\delta k<\Sigma_{\chi}^{2} \tau_{c}$ which contradicts $\omega \gtrsim 1 / \tau_{c}$ as was argued beneath Eq. (35). It actually also contradicts Eq. (10). In the later case, using $c_{3}+3 c_{2} \sim-\omega \tau_{c}$ again implies the contradictory condition $\delta k<\Sigma_{\chi}^{2} \tau_{c}$.

Thus, the underdamped modes of a well conducting chiral matter are stable. The dissipation beats the growth. 


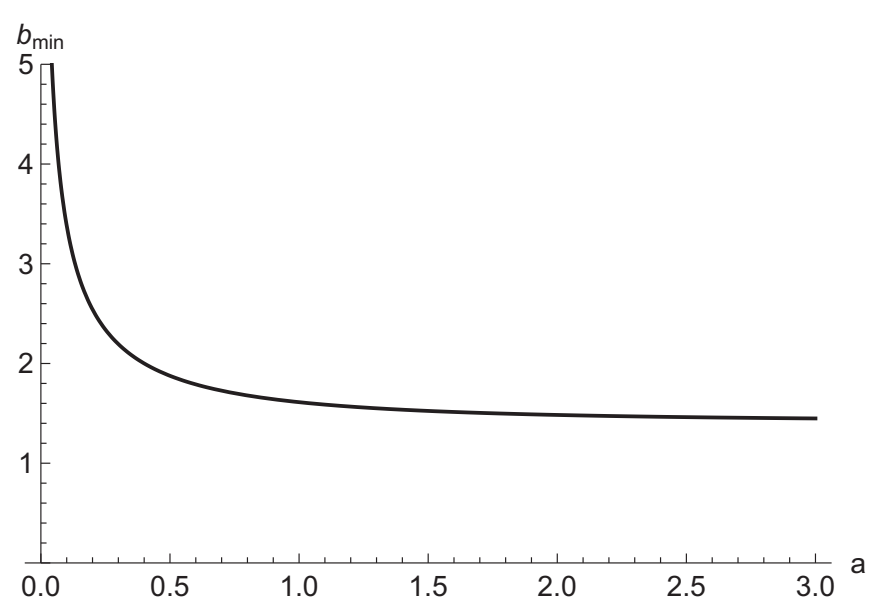

FIG. 1. The minimum values of $b=\Sigma_{\chi} / \sigma$ at given $a=\sigma \tau_{c}$ at which the average energy of magnetic field is unstable in a poorly conducting chiral matter.

Turning to the poorly conducting chiral matter we distinguish two cases. (i) If $\omega \tau_{c} \gg 1$, then $c_{3}-c_{2} \sim 1 / \omega \tau_{c}$ and $v_{0} \omega>\sigma$ implies $\delta k^{2}<\Sigma_{\chi}^{2} / \sigma \tau_{c}$ that contradicts Eq. (10). (ii) If $\omega \tau_{c} \ll 1$, then according to Eq. $30 c_{3}-c_{2} \sim \omega \tau_{c}$ and $\delta k<$ $\Sigma_{\chi}^{2} \tau_{c}$. The consistency with Eq. (10) requires that $\sigma \tau_{c}<1$ which is allowed for a poor conductor since $\sigma \tau_{c} \sim e^{2} / g^{4} \ll 1$. Thus we conclude that these modes are unstable, provided that the electrical conductivity is sufficiently small $\sigma<1 / \tau_{c} \sim$ $T g^{4}$.

The unstable modes of average energy read

$\left\langle\mathcal{E}_{\boldsymbol{k} \lambda}\right\rangle=\frac{k}{2} u_{0} e^{\nu_{0} k t-\sigma t}=\frac{k}{2} u_{0} \exp \left\{\frac{\Sigma_{\chi}^{2}}{2 k}\left(c_{3}-c_{2}\right) t-\sigma t\right\}$.

To make a more quantitative estimate it is useful to employ the model (29). Equation (46) reads

$$
\left\langle\mathcal{E}_{\boldsymbol{k} \lambda}\right\rangle=\frac{k}{2} u_{0} \exp \left\{\frac{\Sigma_{\chi}^{2}}{2 k} \frac{2 \omega \tau_{c}}{1+4 \omega^{2} \tau_{c}^{2}} t-\sigma t\right\} .
$$

Denote $y=2 \omega \tau_{c}, a=\sigma \tau_{c}$, and $b=\Sigma_{\chi} / \sigma$. With this notations one can write Eq. (47) as

$$
\left\langle\mathcal{E}_{\boldsymbol{k} \lambda}\right\rangle=\frac{k}{2} u_{0} e^{f(y) \sigma t}, \quad f(y)=\frac{b^{2} y}{\sqrt{y^{2} / a^{2}+1}\left(1+y^{2}\right)}-1 .
$$

The expression in the exponent has a maximum

$$
f\left(y_{0}\right)=\frac{4 \sqrt{2} a b^{2}}{\left[4+a\left(-a+\sqrt{8+a^{2}}\right)\right] \sqrt{2+a\left(a+\sqrt{8+a^{2}}\right)}}-1
$$

at $y_{0}=\frac{1}{2} \sqrt{-a^{2}+a \sqrt{8+a^{2}}}$. The instability develops only if $f\left(y_{0}\right)>0$ which gives the minimal value of $b$ as a function of $a$

$$
b_{\min }=a^{-1 / 2}\left(1+y_{0}^{2}\right)^{1 / 2}\left(1+a^{2}+2 y_{0}^{2}\right)^{1 / 4}
$$

shown in Fig. 1. $f\left(y_{0}\right)$ monotonically increases with $a$ reaching $b^{2} / 2-1$ at $a \rightarrow \infty$. Thus, at large $a$ the condition for instability is $b^{2}>2$.

For example, consider the quark-gluon plasma produced in heavy-ion collisions. It has the electrical conductivity $\sigma \approx$ $5 \mathrm{MeV}$, and the correlation time $\tau_{c} \approx 5 \mathrm{fm}$ so that $a=0.12$. From $f\left(y_{0}\right)=0$ in Eq. (49) one can find that the corresponding $b_{\min }=3.1$. Thus, the instability in the magnetic field energy occurs if the chiral conductivity dispersion is at least of the order of $\Sigma_{\chi}=15 \mathrm{MeV}$.

Plugging Eq. (45) into Eq. (42) one finds that the average magnetic helicity of each mode has the same instability as the average energy

$$
\left\langle\mathcal{H}_{k \lambda}\right\rangle=\frac{\lambda u_{0}}{2} e^{\nu_{0} k t-\sigma t}
$$

However, since $v_{0}$ is proportional to $\alpha^{2}$ and hence helicity independent, the total average magnetic helicity of the magnetic field (40) vanishes identically

$$
\langle\mathcal{H}\rangle=0 .
$$

To be sure, this equation does not preclude the magnetic helicity fluctuations. It only indicates that the contributions of the opposite helicity states to the magnetic helicity are on average of equal magnitude and have opposite sign.

\section{B. The overdamped modes $k<\sigma / 2$}

In the overdamped case $\omega^{2}<0$, a possible instability may comes about if $\operatorname{Re}\left(\nu_{-} \omega\right)>\sigma$. However, expanding at $k \ll$ $\sigma / 2$ one finds $\nu_{-} \omega-\sigma=-\alpha^{2} c_{1} \sigma / 4-2 k^{2} / \sigma$ plus imaginary terms. Thus, the overdamped modes are stable.

\section{The critically damped mode $k=\sigma / 2$}

Finally, consider the critically damped mode. As explained in Sec. II B the corresponding equations can be obtained from Eqs. (43) and (45) be taking $\omega \rightarrow \beta$ and $\alpha \rightarrow 1$. In this case $\nu_{0}|\beta|-\sigma \sim|\beta|^{2} \tau_{c}-\sigma \sim\left(\Sigma_{\chi} \tau_{c}-1\right) \sigma$, where Eqs. 30 and (19) were used. However, $\Sigma_{\chi} \tau_{c} \ll 1$. Thus, the critical mode is stable.

\section{Conclusion}

It has been argued in this section that the average field energy becomes unstable and grows exponentially if the chiral matter has small enough electrical conductivity $\sigma<g^{4} T$ and large dispersion of the chiral conductivity $\Sigma_{\chi} \gg \sigma$. The more precise expression is given by Eq. (50). The average magnetic helicity vanishes.

\section{SUMMARY AND DISCUSSION}

This paper addresses the time-evolution of the magnetic field in hot nuclear matter at times much longer than the sphaleron transition time $\tau_{c}$. In this limit the chiral conductivity $\sigma_{\chi}$ is treated as the random process. By expanding the electromagnetic field in the basis of the circularly polarized waves it is shown that the field amplitudes obey the stochastic harmonic oscillator equation. The relevant observable 
quantities are statistical averages such as the average magnetic field and average magnetic field energy.

Using the method of [33] the equations for the timeevolution of the average amplitude and its second moments were derived and solved. The solutions indicate that the average magnetic field is exponentially damped, see Eq. (12). The time-evolution of the average field energy is more complicated and depends on the electrical conductivity of the chiral matter. At high temperatures when the quark-gluon plasma is expected to be highly conductive, the dissipation effects prevent the development of instabilities. However, at temperatures higher but comparable to the critical temperature, the quarkgluon plasma is known to be a poor electric conductor. It is argued that if the electrical conductivity satisfies $\sigma<g^{4} T$, the average field energy is unstable, provided that the dispersion of the chiral conductivity fulfills the condition $\Sigma_{\chi} \gg \sigma$. The corresponding unstable modes are $k>\sigma / 2$. A more accurate condition is derived in Sec. IV A.

The magnetic helicity of the right and left-handed modes also increases exponentially. However, the total magnetic helicity vanishes identically. This implies that the conservation of the total helicity cannot tame the chiral instability at later times as it does at early times [4,17,28,29]. Combined with the fact that the growth rate of the average field energy is independent of $\lambda$ indicates that this instability is not chiral.

These results are in a striking contrast with the constant $\sigma_{\chi}$ approximation which is valid at early times $t \ll \tau_{c}$ when the sphaleron transitions can be neglected. In that case, the magnetic field itself is unstable and the unstable modes are soft $k<\sigma_{\chi}$. Moreover, the instability is chiral as it occurs for only one of the helicity modes at a time depending on the sign of $\sigma_{\chi}$.

\section{ACKNOWLEDGMENTS}

I wish to thank Alex Travesset for an informative discussion. This work was supported in part by the US Department of Energy under Grant No. DE-FG02-87ER40371.

\section{APPENDIX: ELECTRODYNAMICS IN HOT NUCLEAR MATTER WITH CP-ODD DOMAINS}

The $C P$-odd domains in the chiral matter can be described by a pseudoscalar field $\theta$ whose interactions with the electromagnetic $F_{\mu \nu}$ and color $G_{\mu \nu}^{a}$ fields are governed by the Lagrangian [21-23]

$$
\begin{aligned}
\mathcal{L}= & \mathcal{L}_{\mathrm{QED}}+\mathcal{L}_{\mathrm{QCD}}-\frac{c_{A}}{4} \theta F_{\mu \nu} \tilde{F}^{\mu \nu}-\frac{c_{A}^{\prime}}{4} \theta G_{\mu \nu}^{a} \tilde{G}^{a \mu \nu} \\
& +f^{2}\left[\frac{1}{2}\left(\partial_{\mu} \theta\right)^{2}-\frac{1}{2} m_{\mathrm{ax}}^{2} \theta^{2}\right]
\end{aligned}
$$

where $\tilde{F}_{\mu \nu}=\frac{1}{2} \epsilon_{\mu \nu \lambda \rho} F^{\lambda \rho}$ is the dual field tensor, $c_{A}, c_{A}^{\prime}$ are the QED and QCD anomaly coefficients respectively and $f, m_{\mathrm{ax}}$ are constants with mass dimension one. The corresponding equation of motion of the $\theta$-field is

$$
\left(\partial^{2}+m_{\mathrm{ax}}^{2}\right) \theta=-\frac{1}{4 f^{2}}\left(c_{A}^{\prime} G_{\mu \nu}^{a} \tilde{G}^{a \mu \nu}+c_{A} F_{\mu \nu} \tilde{F}^{\mu \nu}\right) .
$$

In the hot nuclear matter the electromagnetic contribution to the topological charge density is presumed to be negligible so that the $\theta$-field dynamics is driven primarily by the topologically non-trivial gluon configurations. The equations of motion of electromagnetic field read

$$
\begin{aligned}
& \partial_{\mu} F^{\mu v}=j^{v}-c_{A} \tilde{F}^{\mu \nu} \partial_{\mu} \theta, \\
& \partial_{\mu} \tilde{F}^{\mu \nu}=0 .
\end{aligned}
$$

In the radiation gauge they yield Eq. (1) where the first derivatives $\partial^{\mu} \theta$ of the slowly varying field $\theta$ are replaced by their constant domain-average values, in particular $\sigma_{\chi}=c_{A} \dot{\theta}$ [39].
[1] D. E. Kharzeev, L. D. McLerran, and H. J. Warringa, The effects of topological charge change in heavy ion collisions: 'Event by event P and CP violation', Nucl. Phys. A 803, 227 (2008)

[2] K. Fukushima, D. E. Kharzeev, and H. J. Warringa, The chiral magnetic effect, Phys. Rev. D 78, 074033 (2008)

[3] F. D. M. Haldane, Berry Curvature on the Fermi Surface: Anomalous Hall Effect as a Topological Fermi-Liquid Property, Phys. Rev. Lett. 93, 206602 (2004).

[4] A. Boyarsky, J. Frohlich, and O. Ruchayskiy, Self-Consistent Evolution of Magnetic Fields and Chiral Asymmetry in the Early Universe, Phys. Rev. Lett. 108, 031301 (2012).

[5] K. Tuchin, Electromagnetic field and the chiral magnetic effect in the quark-gluon plasma, Phys. Rev. C 91, 064902 (2015).

[6] C. Manuel and J. M. Torres-Rincon, Dynamical evolution of the chiral magnetic effect: Applications to the quark-gluon plasma, Phys. Rev. D 92, 074018 (2015).

[7] M. Joyce and M. E. Shaposhnikov, Primordial Magnetic Fields, Right-Handed Electrons, and the Abelian Anomaly, Phys. Rev. Lett. 79, 1193 (1997).
[8] D. E. Kharzeev, The chiral magnetic effect and anomalyinduced transport, Prog. Part. Nucl. Phys. 75, 133 (2014).

[9] Z. V. Khaidukov, V. P. Kirilin, A. V. Sadofyev, and V. I. Zakharov, On magnetostatics of chiral media, arXiv:1307.0138.

[10] V. P. Kirilin, A. V. Sadofyev, and V. I. Zakharov, Anomaly and long-range forces, arXiv:1312.0895.

[11] A. Avdoshkin, V. P. Kirilin, A. V. Sadofyev, and V. I. Zakharov, On consistency of hydrodynamic approximation for chiral media, Phys. Lett. B 755, 1 (2016).

[12] Y. Akamatsu and N. Yamamoto, Chiral Plasma Instabilities, Phys. Rev. Lett. 111, 052002 (2013).

[13] M. Dvornikov and V. B. Semikoz, Magnetic field instability in a neutron star driven by the electroweak electron-nucleon interaction versus the chiral magnetic effect, Phys. Rev. D 91, 061301(R) (2015).

[14] P. V. Buividovich and M. V. Ulybyshev, Numerical study of chiral plasma instability within the classical statistical field theory approach, Phys. Rev. D 94, 025009 (2016) 
[15] G. Sigl and N. Leite, Chiral magnetic effect in protoneutron stars and magnetic field spectral evolution, J. Cosmol. Astropart. Phys. 01 (2016) 025.

[16] X.-1. Xia, H. Qin, and Q. Wang, Approach to ChandrasekharKendall-Woltjer state in a chiral plasma, Phys. Rev. D 94, 054042 (2016).

[17] D. B. Kaplan, S. Reddy, and S. Sen, Energy conservation and the chiral magnetic effect, Phys. Rev. D 96, 016008 (2017).

[18] V. P. Kirilin and A. V. Sadofyev, Anomalous transport and generalized axial charge, Phys. Rev. D 96, 016019 (2017).

[19] M. Mace, N. Mueller, S. Schlichting, and S. Sharma, Chiral Instabilities and the Onset of Chiral Turbulence in QED Plasmas, Phys. Rev. Lett. 124, 191604 (2020).

[20] D. Biskamp, Nonlinear Magnetohydrodynamics (Cambridge University Press, Cambridge, 1993).

[21] F. Wilczek, Two Applications of Axion Electrodynamics, Phys. Rev. Lett. 58, 1799 (1987).

[22] S. M. Carroll, G. B. Field, and R. Jackiw, Limits on a Lorentz and parity violating modification of electrodynamics, Phys. Rev. D 41, 1231 (1990).

[23] P. Sikivie, On the interaction of magnetic monopoles with axionic domain walls, Phys. Lett. B 137, 353 (1984).

[24] D. E. Kharzeev and H. J. Warringa, Chiral magnetic conductivity, Phys. Rev. D 80, 034028 (2009).

[25] X. G. Huang, Electromagnetic fields and anomalous transports in heavy-ion collisions-A pedagogical review, Rep. Prog. Phys. 79, 076302 (2016).

[26] S. L. Adler, Axial vector vertex in spinor electrodynamics, Phys. Rev. 177, 2426 (1969).

[27] J. S. Bell and R. Jackiw, A PCAC puzzle: $\pi_{0} \rightarrow \gamma \gamma$ in the sigma model, Nuovo Cim. A 60, 47 (1969).
[28] Y. Hirono, D. E. Kharzeev, and Y. Yin, Self-similar inverse cascade of magnetic helicity driven by the chiral anomaly, Phys. Rev. D 92, 125031 (2015).

[29] K. Tuchin, Taming instability of magnetic field in chiral medium, Nucl. Phys. A 969, 1 (2018).

[30] P. B. Arnold, D. Son, and L. G. Yaffe, The hot baryon violation rate is $\mathrm{O}\left(\alpha_{\mathrm{w}}^{5} \mathrm{~T}^{4}\right)$, Phys. Rev. D 55, 6264 (1997).

[31] D. Bodeker, On the effective dynamics of soft non-Abelian gauge fields at finite temperature, Phys. Lett. B 426, 351 (1998).

[32] P. B. Arnold, D. T. Son, and L. G. Yaffe, Effective dynamics of hot, soft non-Abelian gauge fields. Color conductivity and $\log$ (1/alpha) effects, Phys. Rev. D 59, 105020 (1999).

[33] N. G. Van Kampen, Stochastic differential equations, Phys. Rept. 24, 173 (1976).

[34] P. B. Arnold, G. D. Moore, and L. G. Yaffe, Transport coefficients in high temperature gauge theories. 1. Leading log results, J. High Energy Phys. 11 (2000) 001.

[35] G. Aarts, C. Allton, J. Foley, S. Hands, and S. Kim, Spectral Functions at Small Energies and the Electrical Conductivity in Hot, Quenched Lattice QCD, Phys. Rev. Lett. 99, 022002 (2007).

[36] H.-T. Ding, A. Francis, O. Kaczmarek, F. Karsch, E. Laermann, and W. Soeldner, Thermal dilepton rate and electrical conductivity: An analysis of vector current correlation functions in quenched lattice QCD, Phys. Rev. D 83, 034504 (2011).

[37] W. Cassing, O. Linnyk, T. Steinert, and V. Ozvenchuk, On the Electric Conductivity of Hot QCD Matter, Phys. Rev. Lett. 110, 182301 (2013).

[38] Y. Yin, Electrical conductivity of the quark-gluon plasma and soft photon spectrum in heavy-ion collisions, Phys. Rev. C 90, 044903 (2014).

[39] K. Tuchin, Photon radiation in hot nuclear matter by means of chiral anomalies, Phys. Rev. C 99, 064907 (2019). 